\title{
Mythos Kostenerstattung in der GKV - Mehr Transparenz, mehr Kostenbewusstsein, weniger Inanspruchnahme?
}

Stefan Greß

Ingo Heberlein

Stephanie Heinemann

Dea Niebuhr

Prof. Dr. Stefan Greß ist Professor für Versorgungsforschung und

Gesundheitsökonomie am

Fachbereich Pflege und

Gesundheit der Hochschule

Fulda

Prof. Ingo Heberlein ist Professor für Sozial- und Gesundheitsrecht am Fachbereich Sozial- und

Kulturwissenschaften der

Hochschule Fulda

Stephanie Heinemann ist Wissenschaftliche Mitarbeiterin am Fachbereich Pflege und Gesundheit der Hochschule Fulda

Prof. Dea Niebuhr ist Professorin für Health Technology Assessment und Gesundheitssystemdesign am Fachbereich Pflege und

Gesundheit der Hochschule Fulda

\begin{abstract}
Vor dem Hintergrund des im Koalitionsvertrag beschlossenen Ausbaus der Kostenerstattung in der gesetzlichen Krankenversicherung (GKV) ist nicht auszuschließen, dass die Bundesregierung während der laufenden Legislaturperiode weitere Maßnahmen beschließen wird, um Kostenerstattung für die Versicherten attraktiver zu machen. Allein durch die Einführung des Kostenerstattungsprinzips in der GKV ist eine Zunahme der Steuerungskompetenz des Patienten jedoch nicht zu erwarten. Die Patienten sind nicht in der Lage, durch diesen Zugewinn an reinen Preisinformationen angebotsinduzierte Nachfrage zu verhindern. Es gibt eher Anzeichen dafür, dass ein steigendes Kostenbewusstsein im Rahmen eines ökonomisch rationalen Verhaltens zu steigender Inanspruchnahme gesundheitlicher Leistungen führen würde.
\end{abstract}

\section{Einleitung}

In der gesetzlichen Krankenversicherung (GKV) erhalten die Versicherten im Gegensatz zur privaten Krankenversicherung (PKV) grundsätzlich Leistungen nach dem Sachleistungsprinzip. Der Anteil der Versicherten, die einen Kostenerstattungstarif in der GKV gewählt haben, liegt deutlich unter einem Prozent (GKVSpitzenverband 2009). Diesen Zustand will die Bundesregierung aus CDU/CSU und FDP gemäß der Vereinbarungen im Koalitionsvertrag ändern: „Wir wollen die Transparenz für Ärztinnen und Ärzte sowie Versicherte erhöhen. Deshalb wollen wir die Möglichkeiten der Kostenerstattung ausweiten. Es dürfen dem Versicherten durch die Wahl der Kostenerstattung keine zusätzlichen Kosten entstehen (CDU et al. 2010: 88). “ Diese Absicht hat ihren vorläufigen Kulminationspunkt in den Regelungen des GKV-Finanzierungsgesetzes (GKVFinG) zur Kostenerstattung gefunden.
In diesem Beitrag werden zunächst die potenziellen Vorteile der Kostenerstattung gesundheitsökonomisch analysiert. Anschließend werden internationale Erfahrungen mit einem Parallelsystem aus Kostenerstattungs- und Sachleistungsprinzip in Australien und den Niederlanden sowie die Regelungen des GKV-Finanzierungsgesetzes diskutiert. Abschließend werden drei Szenarien für den Ausbau der Kostenerstattung in der GKV kritisch bewertet.

\section{Kostenerstattung in der gesund- heitsökonomischen Theorie}

Die Unterschiede zwischen Sachleistungs- und Kostenerstattungsprinzip liegen im Dreiecksverhältnis zwischen Arzt,

1 Dieser Beitrag stellt eine Kurzfassung eines vom Fachbereich Pflege und Gesundheit der Hochschule Fulda veröffentlichten Diskussionspapiers dar (Greß et al. 2011). 
Patienten und Krankenkassen. Bei Einführung der Kostenerstattung verändern sich die Zahlungsströme. Die Patienten treten bei der Bezahlung der vom Arzt in Rechnung gestellten Leistungen zunächst in Vorkasse, denn parallel müssen die $\mathrm{Pa}$ tienten versuchen, ihre Auslagen von ihrer Krankenkasse erstattet zu bekommen. Zumindest bei einer vollständigen Umstellung vom Sachleistungs- auf das Kostenerstattungsprinzip fallen außerdem die einschlägigen vertraglichen Regelungen zwischen Krankenkassen und Kassenärztlichen Vereinigungen weg.

Auf den ersten Blick ergeben sich durch die Rechnungsstellung des Arztes und den zwischen Arzt und Patient entstehenden Zahlungsstrom drei zentrale Vorteile. Erstens bekäme der Patient bzw. der Versicherte durch die Rechnungsstellung des Arztes einen Überblick über Art, Menge und Kosten der erbrachten und abgerechneten Leistungen. Diese Transparenz wiederum wird als wesentliche Voraussetzung für den Abbau des Informationsgefälles zwischen dem Arzt und dem Patienten angesehen. Darüber hinaus könnte der Patient durch die Überprüfung der Rechnungen Abrechnungsbetrug wirkungsvoll verhindern (Schäfer 2008).

Der zweite entscheidende Vorteil des Kostenerstattungsprinzips wird darin gesehen, dass durch die Veränderung der Zahlungsströme wirksame Anreize für eine zurückhaltende Inanspruchnahme von Gesundheitsleistungen gesetzt würden. Die Veränderung der Zahlungsströme würde damit nicht nur die Transparenz für die Versicherten erhöhen, sondern als Konsequenz auch das Inanspruchnahmeverhalten verändern. Kostentransparenz sei demnach eine wichtige Voraussetzung dafür, dass sich Patienten kostenbewusst verhalten (Schäfer 2008).

Drittens wird erwartet, dass Versicherte und Patienten alleine durch die Veränderung der Zahlungsströme zunehmend aktiv Steuerungsverantwortung übernehmen und Anreize zur angebotsinduzierten Nachfrage auf ärztlicher Seite konterkarieren können. Kostenerstattung ermögliche danach mehr Transparenz über das Leistungsgeschehen, fördere das Kostenbewusstsein bei allen Beteiligten und sei eine wichtige Voraussetzung für eine gezieltere Inanspruchnahme medizinischer Leistungen (Bundesministerium für Gesundheit/Bundesärztekammer 2010).
In der Gesundheitsökonomie wird in der Regel davon ausgegangen, dass die Verhaltensannahmen für den nutzenmaximierenden Homo oeconomicus aus der neoklassischen Mikroökonomie auch im Verhältnis zwischen Arzt und Patient bzw. im Verhältnis zwischen Versicherten und Krankenkasse gelten. Die Patienten nehmen Gesundheitsleistungen in Anspruch, solange sie sich hiervon noch einen positiven Zusatznutzen versprechen. Die Versicherten in der gesetzlichen Krankenversicherung verfügen darüber hinaus in der ambulanten ärztlichen Versorgung abgesehen von der Praxisgebühr - über einen Vollversicherungsschutz. Die Inanspruchnahme von zusätzlichen Leistungen ist damit nicht mit zusätzlichen Kosten verbunden. Dies führt dazu, dass $\mathrm{Pa}$ tienten mit vollständigem Krankenversicherungsschutz mehr Leistungen in Anspruch nehmen, als dies ohne oder mit nur partiellem Krankenversicherungsschutz der Fall wäre (Wasem/Greß 2004).

An diesen Anreizen würde sich für die Versicherten jedoch durch die Einführung des Kostenerstattungsprinzips nichts ändern. In der einschlägigen Literatur wird sogar gemutmaßt, dass die höhere Kostentransparenz zu einer Ausweitung des Konsums führen würde. Versicherte könnten sich dazu veranlasst sehen, ihre gezahlten Krankenversicherungsbeiträge und die abgerechneten Leistungen gegenüberzustellen. Bei einem negativen Saldo - mehr gezahlte Beiträge als abgerechnete Leistungen - könnten damit sogar Anreize zur Leistungsausweitung entstehen (Wasem 2000).

Durch die Ausweitung des Kostenerstattungsprinzips wird eine Zunahme der Steuerungskompetenz des Patienten erwartet. Die Argumentationskette „mehr Transparenz - mehr Kostenbewusstsein - mehr Wirtschaftlichkeit" setzt voraus, dass sich Arzt und Patient als weitgehend gleichberechtigte Partner gegenübertreten. Nur wenn beide Seiten den Nutzen und die Angemessenheit einer Behandlung richtig einschätzen können, wird der Spielraum für die Erbringung angebotsinduzierter Leistungen sinken und die Wirtschaftlichkeit der Leistungserbringung steigen (Jacobs et al. 2010).

Das Informationsgefälle zwischen Arzt und Patient hat seine Ursache jedoch vor allem in unterschiedlichen Kenntnissen hinsichtlich der angemessenen Diagnostik und Wahl der angemessenen Therapie von Erkrankungen (Rice 2003). Ein Informationszuwachs für den Patienten hinsichtlich Art, Menge und Kosten der abgerechneten Leistungen ändert an diesem Informationsgefälle und dem strukturellen Ungleichgewicht in der Beziehung zwischen Arzt und Patient nichts (Wasem 2000). Aus der Rechnung des Arztes kann der Patient keine Informationen über die Angemessenheit oder gar 
scheinen sich hier für die Versicherten ausgeprägte Wahlmöglichkeiten zu ergeben.

Vordergründig scheint es im niederländischen Krankenversicherungssystem dann auch eine Tendenz zum Kostenerstattungsprinzip zu geben. Der Anteil der Versicherten mit einem Sachleistungstarif ist seit dem Jahr 2006 von 67 Prozent auf 50 Prozent zurückgegangen. Im gleichen Zeitraum hat es einen deutlichen Anstieg von Tarifen gegeben, die einen Mix aus Kostenerstattung und Sachleistung vorsehen (Vektis 2009).

Diese Verschiebung ist jedoch nicht aufgrund aktiver Wahlentscheidungen der Versicherten zustande gekommen, sondern durch einen einseitig von den Krankenversicherern vorgenommenen Tarifwechsel (van de Ven et al. 2009). Dieser Tarifwechsel wurde von den Versicherten in der Regel nicht beanstandet, weil die Versicherten den Unterschied zwischen Kostenerstattung und Sachleistung normalerweise nicht kennen. Niederländische Patienten mit KostenerstattungstaAus rif müssen nicht in Vorkasse treten. Außerdem können die Ärzte im Kostenerstattungstarif keine erhöhten Sätze abrechnen. Der Ärzteschaft entsteht damit kein finanzieller Vorteil durch die Behandlung von Patienten mit Kostenerstattungstarif.

Die wissenschaftliche Evaluation der Krankenversicherungsreform in den Niederlanden kam kürzlich dennoch zu einer eher pessimistischen Bewertung des Kostenerstattungsprinzips. Die Skepsis bezieht sich auf die entstehende Gefährdung des Sicherstellungsauftrags. Beim Angebot von Kostenerstattungstarifen hätten demnach die Versicherten keine Garantie dafür, dass sie im Bedarfsfall die versicherten Leistungen auch tatsächlich erhalten könnten. Im
Extremfall sei eine Situation vorstellbar, in der die Krankenversicherer ausschließlich Kostenerstattungstarife anbieten und sich damit ihrer Verantwortung für die Sicherstellung der gesundheitlichen Versorgung entziehen würden. Dies sei besonders dann problematisch, wenn die Versicherten über die Unterschiede zwischen Sachleistungs- und Kostenerstattungsprinzip nicht informiert seien (van de Ven et al. 2009).

In Australien werden die Kosten der in der ambulanten ärztlichen Versorgung abgerechneten Einzelleistungen entweder nach dem Kostenerstattungs- oder dem Sachleistungsprinzip übernommen. Die für den Patienten vorteilhafte Abrechnung nach dem Sachleistungsprinzip ist jedoch davon abhängig, ob der Leistungserbringer das Sachleistungsprinzip und die damit verbundene Gebührenordnung akzeptiert. Entscheiden sich Allgemein- bzw. Fachärzte für die Kostenerstattung, sind diese nicht an die Gebührenordnung gebunden und können die Honorarhöhe für ihre erbrachten Leistungen frei bestimmen. Durch diese Regelungen sollen Anreize zur Niederlassung in unterversorgten Regionen geschaffen werden. Die Erstattung für die Patienten richtet sich jedoch nach der Gebührenordnung - auch wenn der Arzt nach dem Kostenerstattungsprinzip abrechnet. In diesem Fall müssen die Patienten die Differenz zwischen Abrechnungstarif und Erstattungstarif selbst tragen (DoHA 2010).

Die australische Regierung hat daher in den letzten Jahren einen umfangrei- chen sozialen Ausgleich für Personen bzw. Familien mit niedrigem Einkommen sowie chronisch Erkrankte eingeführt, um die steigenden finanziellen Belastungen für die Patienten begrenzen zu können (Van Gool et al. 2006).

Die Patienten haben keinen Einfluss darauf, ob der behandelnde Arzt nach dem Sachleistungs- oder Kostenerstattungsprinzip abrechnet. Zwar haben die Patienten im Grundsatz die Möglichkeit, einen nach dem Sachleistungsprinzip abrechnenden Allgemeinmediziner zu wählen. Regionale Unterschiede in der Arztdichte schränken diese freie Arztwahl aber ein. Insgesamt sind in Australien deutlich weniger Hausärzte in ländlichen

\section{Tab. 1: Kostenerstattung im internationalen Vergleich}

\begin{tabular}{|c|c|c|c|}
\hline & Deutschland (GKV) & Niederlande & Australien \\
\hline $\begin{array}{l}\text { Anreize zur Wahl von Kosten- } \\
\text { erstattung für Versicherte }\end{array}$ & $\begin{array}{l}\text { Gering (Einerseits: bevorzugte } \\
\text { Behandlung. Andererseits: } \\
\text { Differenz zwischen EBM und } \\
\text { GOÄ muss individuell finan- } \\
\text { ziert werden) }\end{array}$ & $\begin{array}{l}\text { Gering (Unterschiede werden } \\
\text { nicht wahrgenommen) }\end{array}$ & $\begin{array}{l}\text { Gering (Wahlmöglichkeit nur } \\
\text { aufgrund freier Arztwahl, die } \\
\text { aber insbesondere für Allge- } \\
\text { meinärzte in ländlichen } \\
\text { Regionen eingeschränkt ist und } \\
\text { für Fachärzte gar nicht gilt) }\end{array}$ \\
\hline $\begin{array}{l}\text { Anreize zum Angebot von } \\
\text { Kostenerstattung für Leistungs- } \\
\text { erbringer }\end{array}$ & $\begin{array}{l}\text { Hoch (unterschiedliche } \\
\text { Vergütungssysteme) }\end{array}$ & $\begin{array}{l}\text { Gering (einheitliches Vergü- } \\
\text { tungssystem) }\end{array}$ & $\begin{array}{l}\text { Hoch (Honorar im Kostener- } \\
\text { stattungssystem frei bestimm- } \\
\text { bar) }\end{array}$ \\
\hline $\begin{array}{l}\text { Anreize zum Angebot von } \\
\text { Kostenerstattung für } \\
\text { Kostenträger }\end{array}$ & $\begin{array}{l}\text { Gering (erhöhte Verwaltungs- } \\
\text { kosten; Verlust an Steuerungs- } \\
\text { fähigkeit; „PKV-Tarife“ nicht } \\
\text { finanzierbar) }\end{array}$ & $\begin{array}{l}\text { Theoretisch (Gefahr, dass sich } \\
\text { Krankenversicherungen ihrer } \\
\text { Steuerungsverantwortung } \\
\text { entziehen) }\end{array}$ & $\begin{array}{l}\text { Nicht relevant (kein wettbe- } \\
\text { werbliches Krankenversiche- } \\
\text { rungssystem) }\end{array}$ \\
\hline
\end{tabular}

Regionen bzw. entlegenen Gemeinden ansässig. In dicht besiedelten Regionen führt der Preiswettbewerb unter den Allgemeinärzten dazu, dass diese sich stärker am Sachleistungsprinzip orientieren. Tabelle 1 fasst die wesentlichen Erkenntnisse aus dem internationalen Vergleich zusammen.

\section{4. Änderungen im GKV-FinG}

Um die Wahrnehmung des generellen Anspruchs auf Kostenerstattung $\mathbb{S} 13$ Abs. 2 SGB V zu erleichtern, hat der Gesetzgeber im Rahmen des GKV-FinG zwei wesentliche Änderungen vorgenommen. Die erste Änderung betrifft Neuregelun- 
gen für die bisher vorgesehenen Erstattungsabschläge für erhöhte Verwaltungskosten und fehlende Wirtschaftlichkeitsprüfungen. Der Erstattungsabschlag für erhöhte Verwaltungskosten wird auf fünf Prozent begrenzt - mit zusätzlichen Regelungen für die Kostenerstattung im Rahmen der Arzneimittelversorgung. Der Abschlag für fehlende Wirtschaftlichkeitsprüfungen entfällt völlig. Außerdem entfällt der Hinweis, dass Zuzahlungen bei der Erstattung ebenfalls in Abzug zu bringen sind. Letzteres ergibt sich aus der Regelung, dass die Erstattungshöhe in der Kostenerstattung auf das Erstattungsniveau im Sachleistungsprinzip begrenzt ist.

Der Wegfall von Abschlägen für das Fehlen von Wirtschaftlichkeitsprüfungen ist zu kritisieren. Der Patient kann unwirtschaftliches Handeln der Leistungserbringer nur vermuten, hat selbst aber keine Handhabe zur Kontrolle, schon aufgrund der unvollkommenen Kenntnis über die Leistungsqualität.

Wenig sachgerecht ist auch die Begrenzung der Abschläge für Verwaltungskosten auf fünf Prozent des Erstattungsbetrags. Dieser Abschlag deckt den zusätzlichen Verwaltungsaufwand - insbesondere für die Beratung der Versicherten und die Administration der Rechnungen - nicht ab. Für die Krankenkassen wird die Option der Kostenerstattung für die Versicherten auch vor dem Hintergrund der Nullrunde für die Verwaltungskosten damit zunehmend finanziell unattraktiv - zumal hier im Gegensatz zu den Wahltarifen nach $\$ 53$ Abs. 4 SGB V kein Verbot der Quersubventionierung gilt.

Die dritte Änderung betrifft die Verkürzung der Bindungsfrist von einem Jahr auf ein Kalendervierteljahr. Diese solle, so die Begründung des Gesetzgebers, größtmögliche Flexibilität bei der Wahl der Kostenerstattung gewährleisten. Einerseits sinkt dadurch im Sinne der Intention des Gesetzgebers die Schwelle für die Versicherten, die Option zur Kostenerstattung wahrzunehmen. Andererseits erleichtert die verkürzte Bindungsfrist auch die Exit-Möglichkeit für Versicherte, die die Option auf Kostenerstattung wieder aufgeben wollen.

Insgesamt hat der Gesetzgeber nur vorsichtige Änderungen im $\mathbb{S} 13$ Abs. 2 SGB V vorgenommen. Ein Anspruch auf Erstattung für die Versicherten besteht weiterhin höchstens in der Höhe der Vergütung, die die Krankenkasse bei Erbrin-
Tab. 2: Szenarien für den Ausbau des Kostenerstattungsprinzips in der GKV

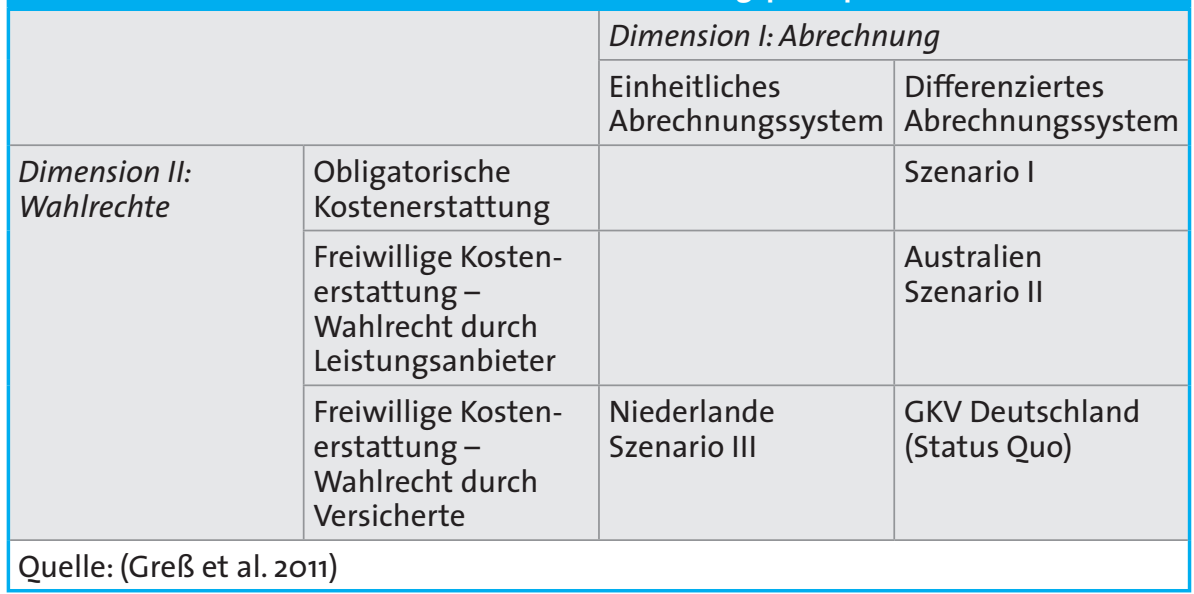

gung als Sachleistung zu erbringen hätte. Eine weitergehende Regelung ohne Angleichung der Vergütungssysteme EBM und GOÄ hätte unabsehbare Konsequenzen für die Ausgabenentwicklung der GKV insbesondere in der ambulanten ärztlichen Versorgung gehabt. Diese Beschränkung des Erstattungsanspruchs ist jedoch das zentrale Element, das die Attraktivität der Kostenerstattungsoption für die Versicherten in der gesetzlichen Krankenversicherung nachhaltig begrenzt. Daher werden die angesprochenen Änderungen im GKV-FinG die Attraktivität der Kostenerstattungsoption nicht wesentlich erhöhen.

Der Gesetzgeber hat im GKV-FinG außerdem zwei zentrale Rahmenbedingun-

gen für Wahltarife generell verändert die Änderungen gelten dementsprechend auch für Wahltarife mit Kostenerstattung. Erstens hat der Gesetzgeber für mehrere Wahltarife - einschließlich derer nach $\mathbb{} 53$ Abs. 4 SGB V - die Mindestbindungsfrist für die Versicherten von drei Jahren auf ein Jahr reduziert. Diese Verkürzung erhöhe nach der Gesetzesbegründung die Wahlfreiheit der Versicherten. Die bisherige Mindestbindungsfrist von drei Jahren für Wahltarife habe die Versicherten davon abgehalten, diese in Anspruch zu nehmen.

Aus Perspektive der Versicherten gilt hier die gleiche ambivalente Bewertung wie bei der Verkürzung der Bindungsfrist der Option zur Kostenerstattung nach $\mathbb{S}$ 13 Abs. 2 SGB V. Einerseits sinkt im Sinne der Intention des Gesetzgebers die
Schwelle für die Versicherten, in einen Kostenerstattungstarif zu wechseln. Andererseits erleichtert die verkürzte Bindungsfrist auch die Exit-Möglichkeit für Versicherte, den Wahltarif nach einem Jahr wieder aufzugeben.
Der von der Bundesregierung beabsichtigte Ausbau des Kostenerstattungsprinzips in der GKV wird mit den beschlossenen Regelungen im GKV-Finanzierungsgesetz nicht gelingen.

Die zweite Änderung besteht in verschärften Nachweispflichten für das Verbot der Quersubventionierung von Wahltarifen zu Lasten der Versichertengemeinschaft. Die Krankenkassen müssen nun der zuständigen Aufsichtsbehörde mindestens alle drei Jahre ein versicherungsmathematisches Gutachten vorlegen, das die wesentlichen Annahmen über die Berechnung der Beiträge und der versicherungstechnischen Rückstellungen offenlegt. Die entsprechende Prüfung hat nach der Gesetzesbegründung durch Aktuare zu erfolgen. Der Gesetzgeber verallgemeinert damit eine als sachgerecht anzusehende Praxis mehrerer Anbieter von Wahltarifen, den Nachweis über die gesetzlichen Anforderungen zur Vermeidung von Quersubventionierungen $\mathrm{zu}$ erbringen. 


\section{Fazit: Szenarien für den Ausbau von Kostenerstattung in der GKV}

Es ist daher davon auszugehen, dass vor dem Hintergrund des im Koalitionsvertrag beschlossenen Ausbaus der Kostenerstattung die Bundesregierung weitere Maßnahmen zum Ausbau der Kostenerstattung beschließen wird. Unter Berücksichtigung der Erfahrungen in Deutschland aber auch in Australien und den Niederlanden stehen hierzu drei Szenarien zur Verfügung (vgl. Tabelle 2). ${ }^{2}$

Szenario I mit obligatorischer Kostenerstattung und unveränderten Abrechnungsmodalitäten würde zu einem erheblichen Anstieg angebotsinduzierter Nachfrage, spürbaren Aufzahlungen der
Versicherten und der Notwendigkeit zu einem sozialen Ausgleich der Mehrbelastungen führen. Weitere Konsequenz wäre außerdem eine Belastung der Versichertengemeinschaft durch steigende Bürokratiekosten und die Finanzierung des sozialen Ausgleichs sowie die Wirkungslosigkeit des Instrumentariums zur Versorgungssteuerung.

Szenario II mit Wahlrecht der Leistungserbringer bei unveränderten $\mathrm{Ab}$ rechnungsmodalitäten würde zu ähnlichen Problemen führen wie die obligatorische Einführung des Kostenerstattungsprinzips. Eine Einschränkung dieses Wahlrechts auf unterversorgte Gebiete würde diese problematischen Auswirkungen lediglich regional begrenzen.
Szenario III mit einem einheitlichen Abrechnungssystem und Wahlrecht für die Versicherten würde eine zentrale Barriere zur Inanspruchnahme des Kostenerstattungsprinzips beseitigen. Zudem würden Anreize zur angebotsinduzierten Nachfrage weitgehend beseitigt. Die Effekte auf die Inanspruchnahme der Versicherten wären dennoch ungewiss. Letztlich ist $\mathrm{zu}$ fragen, ob eine erhöhte Kostentransparenz nicht auch mit geringerem administrativem Aufwand durch eine für die Patienten kostenfreie Patientenquittung erreicht werden könnte.

\footnotetext{
2 Für eine ausführliche Herleitung und Diskussion der Szenarien vgl. (Greß et al. 2011).
}

\section{Literatur:}

Bundesministerium für Gesundheit/Bundesärztekammer (2010). Für eine gerechte und zukunftsfeste Gesundheitsversorgung. Gemeinsame Presseerklärung vom 27.8.2010. Berlin, Bundesministerium für Gesundheit. http://www.bmg.bund.de/SharedDocs/ Pressemitteilungen/DE/2010/pm-10-08-27-gesundheitsversorgung.html (Zugriff am 12. November 2010).

CDU/CSU/FDP (2010). Wachstum. Bildung. Zusammenhalt. Koalitionsvertrag zwischen CDU, CSU und FDP. 17. Legislaturperiode.

DoHA (2010). Medicare Benefits Schedule Book. Operating from 01 November 2010, Stand 1. Mai 2008. Canberra, Australian Government. Department of Health and Ageing http://www.health.gov. au/internet/mbsonline/publishing.nsf/Content/B3424CE2210FF E9FCA2577E6007BFFo8/\$File/201101-MBS.pdf

GKV-Spitzenverband (2009). Bericht des GKV-Spitzenverbandes über die Erfahrungen mit den duch das GKV-WSG bewirkten Rechtsänderungen in $\$ 13$ Abs. 2 SGB V vom 25. März 2009. Berlin, GKV-Spitzenverband.

Greß, S./I. Heberlein/S. Heinemann/D. Niebuhr (2011). Kostenerstattung in der GKV. pg papers 1/2011, Fachbereich Pflege und Gesundheit der Hochschule Fulda.

Jacobs, K./C. Kip/S. Schulze (2010). »Mehr Kostenerstattung in der GKV? Auswirkungen auf Kostentransparenz und Inanspruchnah- meverhalten - Wunsch und Wirklichkeit." Gesundheit und Gesellschaft Wissenschaft 10(4): 17-26.

Rice, T. (2003). The Economics of Health Reconsidered. Second Edition. Chicago, Health Administration Press.

Schäfer, W. (2008). Die Zukunft gehört der Kostenerstattung. Kostenerstattung in der gesetzlichen Krankenversicherung. Eine Bestandsaufnahme. Verband der privatärztlichen Abrechnungsstellen. Berlin, Schriftenreihe PVS-Verband. Bd. 7: 63-84.

van de Ven, W. P. M. M./F. T. Schut/H. E. G. M. Hermans/J. D. de Jong/M. van der Maat/R. Coppen/P. P. Groenewegen/R. D. Friele (2009). Evaluatie Zorgverzekeringswet en Wet op de zorgtoeslag. Den Haag, ZonMW. Programma evaluatie regelgeving deel 27.

Vektis (2009). Jaarcijfers 2009 - Zorgverzekerden. Zeist, Vektis.

Wasem, J. (2000). »Kostenerstattung und Sachleistung aus ökonomischer Sicht.« Medizinrecht(10): 472-77.

Wasem, J./S. Greß (2004). Anreize für die Versicherten in der Gesetzlichen Krankenversicherung - die Diskussion um das »Moral Hazard «-Problem in der GKV. Schriftenreihe des Deutschen Sozialrechtsverbandes, Band 52. Wiesbaden, Verlag Chmielorz: $115-26$. 